\title{
Transformations homéotiques chez la souris provoquées par l'inactivation de I'homéogène Hox-3.1
}

\section{Hervé Le Mouellic Yvan Lallemand Philippe Brûlet}

\section{ADRESSES}

H. Le Mouellic : chargé de recherche à l'Inserm. Y. Lallemand : assistant de recherche de l'Institut Pasteur. P. Brûlet : directeur de recherche au Cnrs. Unité de génétique cellulaire du $\mathrm{Col}-$ lège de France et de l'Institut Pasteur, Cnrs, URA 1148, 25, rue du Doctcur-Roux, 75724 Paris Cedex 15, France.

\section{TIRÉS A PART}

P. Brûlet.

L'utilisation de la recombinaison homologue dans les cellules souches embryonnaires a permis d'obtenir des souris dans lesquelles le gène $H o x-3.1$ est inactivé et remplacé par le gène rapporteur lacZ d'Escherichia coli. Les souris mutantes homozygotes meurent très fréquemment dans les premiers jours après leur naissance. Certaines vertèbres de ces souris sont transformées en prenant l'apparence de vertèbres plus antérieures. Par exemple, une paire de côtes surnuméraires est apparue sur la première vertèbre lombaire. De même les huitièmes côtes, qui sont libres chez les souris sauvages, sont attachées au sternum chez les souris mutantes. La mutation a donc modifié l'identité des cellules embryonnaires qui auraient dû exprimer le gène $H o x-3.1$, comme le font les mutations homéotiques chez la drosophile.

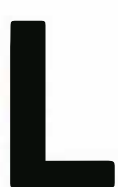

es mécanismes de morphogenèse nécessitent l'établissement préalable des différents axes de l'organisme ainsi que la détermination de coordonnées spatiales le long de ces axes. Ces événements, qui surviennent au cours de l'embryogenèse, permettent aux cellules de connaître leur position dans l'organisme et de suivre un programme spécifique. Par exemple, le squelette des vertébrés est constitué d'os très différents qui sont pourtant formés, selon un programme invariable, à partir de cellules phénotypiquement identiques. La génétique du développement de la mouche Drosophila melanogaster a permis d'identifier de nombreux gènes déterminant l'organisation spatiale de l'embryon [1,2]. Parmi ces différentes classes de gènes, les homéogènes, gènes Hox chez les mammifères et gènes homéotiques chez la drosophile, constituent la famille la plus nombreuse et la mieux conservée au cours de l'évolution [3]. Les analogies portent sur la séquence de ces gènes, caractérisés par la boîte " homéo ", et sur leur organisation en complexe, unique chez la drosophile et en quatre exemplaires dans le génome des mammifères. De plus, l'ordre spatial des domaines d'expression de ces homéogènes dans l'embryon correspond à leur ordonnancement dans les complexes. Le rôle des gènes homéotiques chez la drosophile est d'informer les cellules embryonnaires sur leur position le long de l'axe antéropostérieur et, ainsi, de spécifier l'identité des segments caractérisés par la morphologie de l'épiderme de la larve et de l'adulte. Une mutation 
dans l'un de ces gènes va le plus souvent provoquer la transformation de segments qui vont se développer en prenant l'identité d'autres segments.

L'inactivation d'un homéogène chez la souris est la méthode la plus directe pour savoir si ces gènes sont également responsables de déterminations spatiales dans les embryons de vertébrés. La production de souris mutantes a été récemment rendue possible avec la mutagenèse dirigée par recombinaison homologue et la transgenèse au moyen des cellules souches embryonnaires (ES) [4-6]. La méthode que nous avons développée pour muter l'homéogène Hox-3.1 consiste à remplacer spécifiquement une partie de la phase codante du gène cible par le gène rapporteur lac $Z$ qui code pour l'enzyme $\beta$ galactosidase [7]. Ainsi, le gène Hox-3.1 est non seulement inactivé, mais son expression est remplacée par celle du gène lac $Z$. La révélation de l'activité $\beta$-galactosidase permettra donc de visualiser les cellules qui auraient dû exprimer Hox-3.1 et de suivre leur devenir dans les embryons et les souris portant la mutation à l'état hétérozygote ou homozygote [8].

\section{Remplacement de Hox-3.1 par un gène rapporteur}

L'activité $\beta$-galactosidase peut être révélée in situ en présence d'un substrat spécifique, dont le produit de dégradation donne une coloration bleue. Au cours de l'embryogenèse, l'expression du locus recombiné Hox-3.1-lac $Z$ débute au jour 8,5 après la fécondation, peu après le début de la gastrulation. L'activité $\beta$ galactosidase est alors visible dans toute la partie postérieure de l'embryon et avec la même intensité dans tous les tissus (figure 1). L'organogenèse va s'accompagner de variations importantes dans la distribution de l'activité $\beta$-galactosidase. L'expression de $\operatorname{lac} Z$ va progressivement diminuer dans les régions les plus postérieures de l'embryon. L'activité $\beta$ galactosidase sera également variable selon le tissu considéré, étant particulièrement forte dans le tube neural, moyenne dans les pré-vertèbres et faible dans le rein embryonnaire. L'expression de lac $Z$ va se stabiliser $\mathrm{m} / \mathrm{s} n^{\circ} 4$, vol. 8 , avril 92

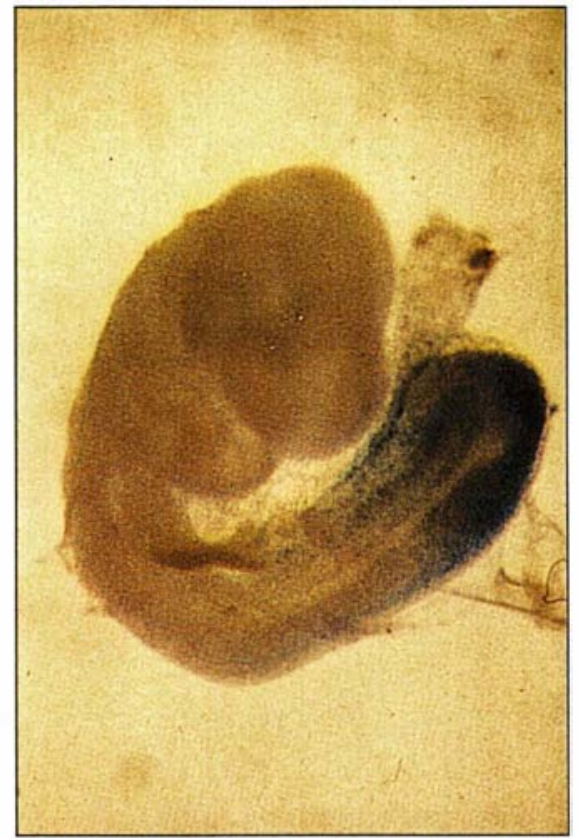

Figure 1. Expression du gène recombiné Hox-3.1-lacz au jour 8,5 de I'embryogenèse. Embryon hétérozygote Hox-3.1+1- marqué in toto en présence de $X$-Gal.

à partir du jour 12,5 de l'embryogenèse, notamment dans les prévertèbres 12 à 21 (figure 2) - qui donneront, respectivement, la $4^{\mathrm{e}}$ vertèbre thoracique (T4) et la $1^{\text {re }}$ vertèbre lombaire (L1). A ce stade, dans le tube neural, l'activité $\beta$-galactosidase est particulièrement élevée dans les cornes ventrales, au niveau des prévertèbres 6 à 14 (6e vertèbre cervicale, C6, à T7), et dans la région médiane, au niveau des pré-vertèbres 3 à 14 (C3 à T7) (figure 2).

L'expression du gène rapporteur lac $Z$ est remarquablement fidèle à la distribution des $A R N m$ du gène Hox-3.1 [9], contrairement aux expériences de transgenèse classique dans lesquelles le nombre et le(s) site(s) d'intégration sont incontrôlables. La révélation de l'activité $\beta$-galactosidase, avec une sensibilité et une résolution supérieures à l'hybridation d'ARNm in situ, a permis de révéler de nouveaux sites d'expression de Hox-3.1 par exemple, dans le squelette des souriceaux. La parfaite identité d'expression de lac $Z$ entre les embryons hétérozygotes Hox-3.1 $1^{+/}$ et homozygotes Hox-3-1-1- montre que la mutation ne semble pas avoir modifié la position des cellules qui auraient dû exprimer $H o x-3.1$.

\section{Transformations en des structures plus antérieures dans le squelette axial des souris mutantes pour le gène Hox-3.1}

L'observation de la colonne vertébrale des mutants Hox-3.1 $1^{-1}$ a révélé la transformation de segments spécifiques qui ont pris l'apparence de segments plus antérieurs. La transformation homéotique la plus visible et la plus postérieure affecte la vertèbre $\mathrm{L} 1$ de tous les homozygotes, sur laquelle s'est développée une $14^{\mathrm{e}}$ paire de côtes (figure 3), alors que les souris sauvages n'en possèdent que 13. Deux autres transformations ont été observées dans la région du sternum chez une forte proportion de mutants homozygotes Hox-3.1 $1^{-1-}$. Chez les animaux sauvages, les extrémités des six premières côtes sont séparées par cinq pièces sternales, ou sternèbres. Les $6^{\mathrm{e}}$ et 7 e côtes sont attachées au même point sur le sternum alors que les $8^{\text {es }}$ côtes sont flottantes. Chez certaines souris Hox-3.1-1-, une sternèbre supplémentaire s'est développée entre les extrémités des $6^{\mathrm{e}}$ et $7^{\mathrm{e}}$ côtes et les $8^{\mathrm{es}}$ se sont attachées au sternum au même point que les $7^{\text {cs }}$ (figure 4). D'autres phénotypes plus subtils, tels que la longueur des côtes ou la morphologie de la vertèbre, nous indiquent la transformation antérieure des vertèbres $\mathrm{T} 10$ et T12. Ainsi, malgré l'absence de critères morphologiques pour tous les segments, il est vraisemblable que chaque vertèbre, de $\mathrm{T} 7$ à L1, ait été transformée en vertèbre plus antérieure. Nous ignorons si les vertèbres antérieures à T6 ou postérieures à L2 ont été affectées par l'inactivation de Hox-3.1. Tous les mutants homozygotes ont montré des transformations qui étaient plus ou moins prononcées et, chez quelques rares hétérozygotes, de faibles transformations pouvaient être également observées. Ces transformations homéotiques ne doivent rien à une éventuelle addition ou duplication de segments, le nombre total de vertèbres étant constant quel que soit le génotype. 

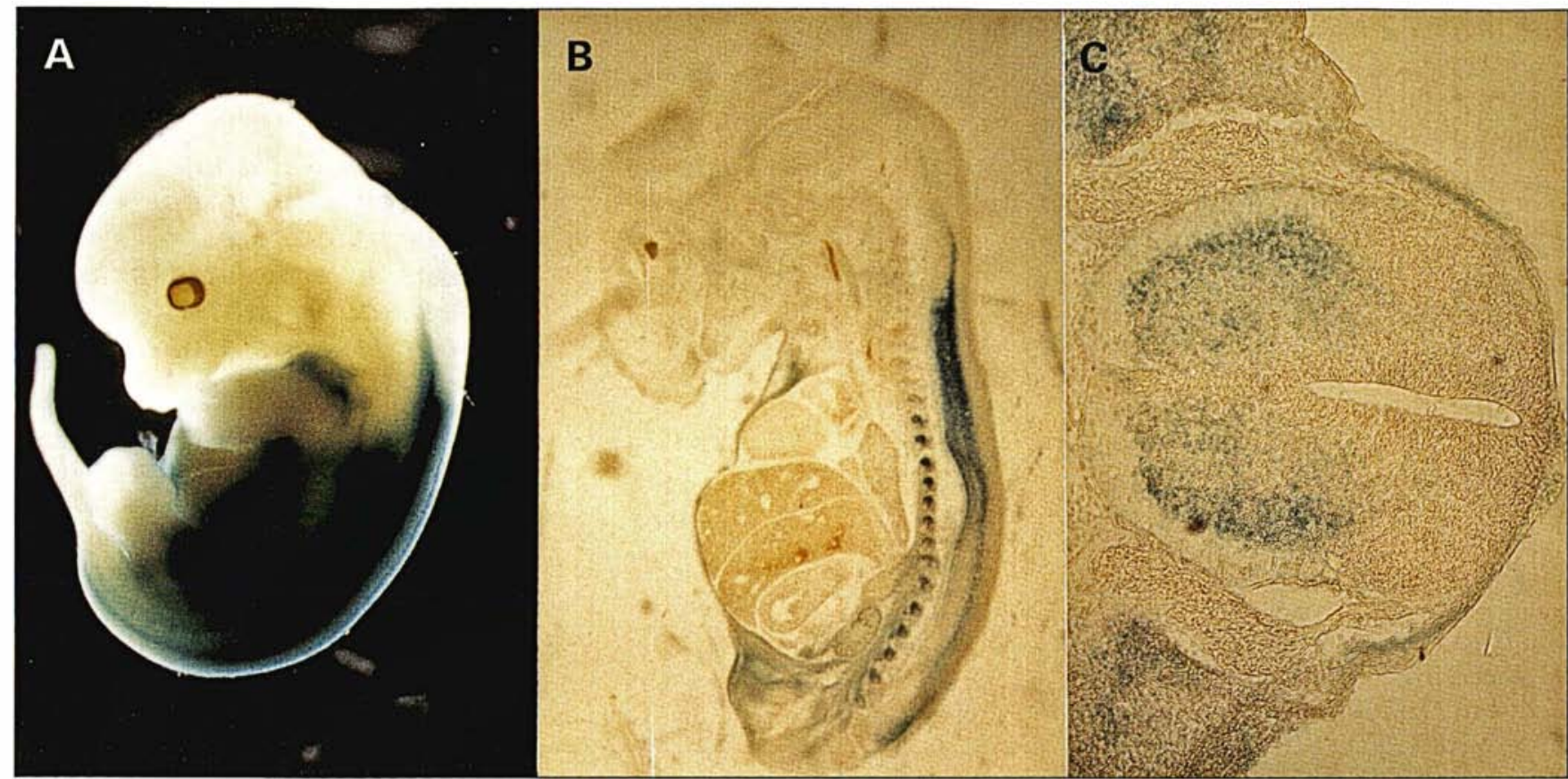

Figure 2. Expression du gène recombiné Hox-3.1-lacZ au jour 12,5 de l'embryogenèse. (A) Embryon hétérozygote Hox-3. $1^{+1-}$ marqué in toto en présence de X-Gal. Coupes parasagittale (B) et transversale (C) d'embryons hétérozygotes révélant l'expression de lacZ dans les cornes ventrales et la région médiane du tube neural, dans l'ectoderme superficiel et dans les prévertèbres. La figure 2B montre également le marquage dans le mésenchyme de la paroi du tronc et dans le rein ainsi que l'absence de marquage dans le cœur, les poumons, le foie ou l'estomac.

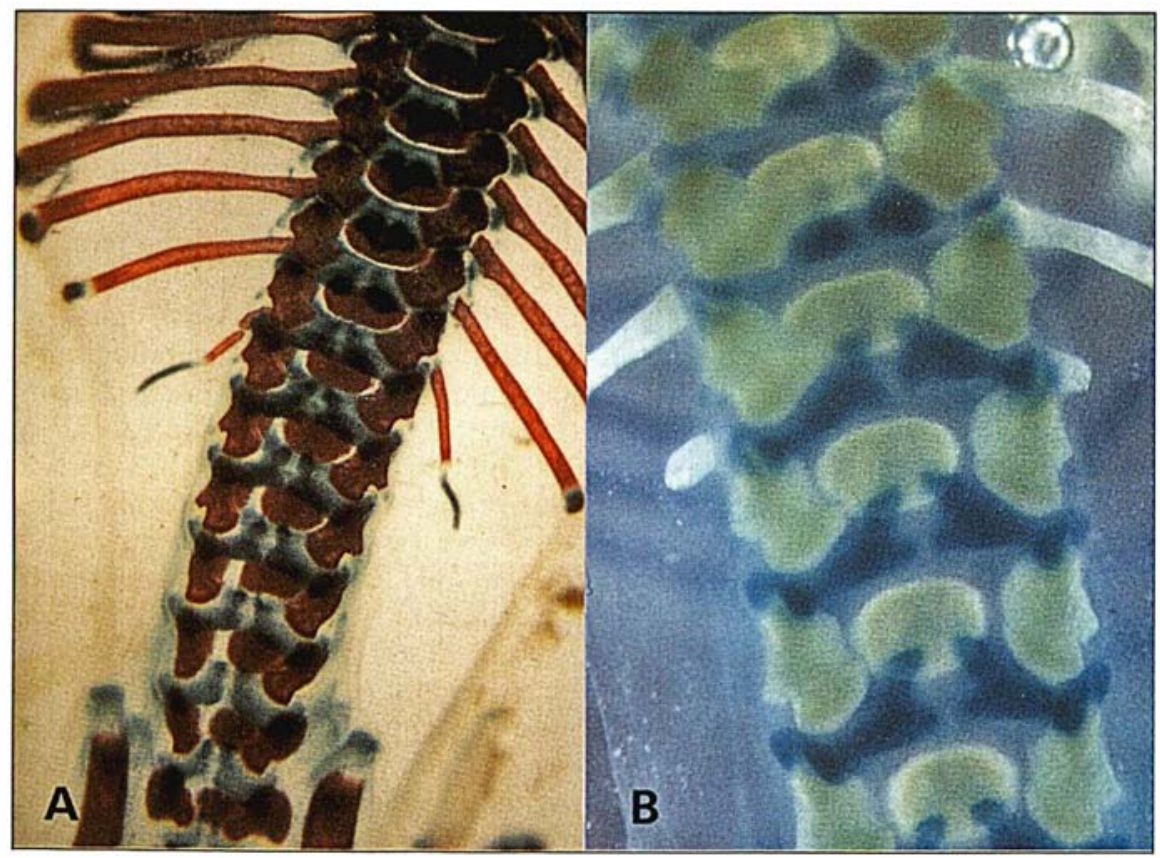

Figure 3. Transformation homéotique de la première vertèbre lombaire (L1). Vertèbre $L 1$ de deux nouveau-nés homozygotes Hox-3.1-1- dont le squelette a été marqué en présence de $X$-Gal. La paire de côtes surnuméraire peut être bien développée (A) ou constituée de bourgeons osseux (B) ou encore asymé. 342

\section{Phénotypes neurologiques des souris homozygotes Hox-3.1 ${ }^{-1-}$}

A la naissance, aucun phénotype particulier ne distingue les descendants de parents $\mathrm{F}_{1}$ hétérozygotes $H o x-3 \cdot 1^{-1-}$. Cependant, seulement $9 \%$ des souris $\mathrm{F}_{2}$ adultes sont homozygotes Hox-3.1 $1^{-1}$. En effet, la plupart des souriceaux mutants homozygotes meurent dans les deux premiers jours après leur naissance alors que leur estomac est visiblement vide de lait. Une hypothermie et une déshydratation seraient certainement consécutives à cette malnutrition que confirme la lente croissance des souris homozygotes survivantes. Ce phénotype pourrait être associé à une anomalie du système orthosympathique, comme le suggère l'expression de Hox-3.1 dans la région des corps cellulaires des neurones sympathiques pré-ganglionnaires. Le système orthosympathique innerve tous les organes vitaux, en opposition avec le système parasympathique, afin de contrôler le métabo- 


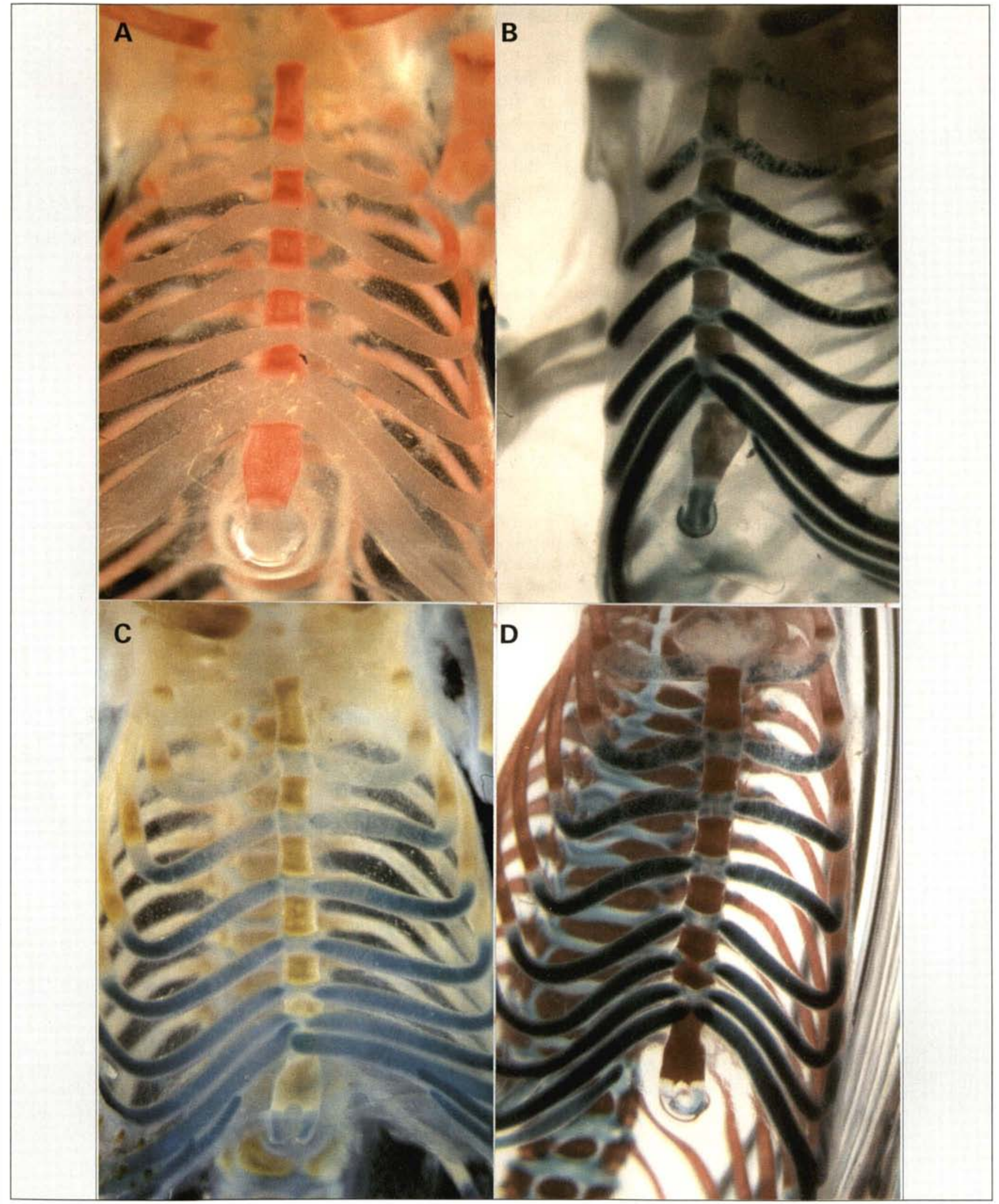

Figure 4. Transformations homéotiques dans la région du sternum. Vues ventrales du sternum de nouveau-nés sauvage Hox-3.1+1+ (A), hétérozygote Hox-3.1+1- (B) ou homozygotes Hox-3.1-1- $(\boldsymbol{C}, \boldsymbol{D})$ dont le squelette a été marqué en présence de $X$-Gal. 


\section{RÉFÉRENCES}

1. Akam M. The molecular basis for metameric pattern in the Drosophila embryo. Development $1987 ; 101: 1-22$.

2. Ingham $\mathbf{P W}$. The molecular genetics of embryonic pattern formation in Drosophila. Nature 1988 ; 335 : 25-34, 744.

3. Duboule D, Dollé $P$, Gaunt SJ. Les gènes du développement des mammifères. $L a$ Recherche 1990 ; 21 : 294-303.

4. Capecchi MR. The new mouse genetics altering the genome by gene targeting. Trends Genet 1989 ; 5 : 70-6.

5. Rossant J, Joyner AL. Towards a molecular-genetic analysis of mammalian development. Trends Genet 1989 ; 5 : 277-83.

6. Babinet C. Les cellules souches embryonnaires de souris : une voic privilégiéc de transformation génćtique à l'échelle de l'animal. médecine/sciences $1992 ; 8: 268-75$

7. Le Moucllic H, Lallemand Y, Brûlet P. Targeted replacement of the homeobox gene Hox-3.1 by the Escherichia coli lac $Z$ in mouse chimeric embryos. Proc Natl Acad Sci USA $1990 ; 87: 4712-6$

8. Le Moucllic H, Lallemand Y, Brûlet P. Homeosis in the mousc induced by a null mutation in the homeogene Hox-3.1. Cell 1992 ; 69 (sous presse).

9. Le Moucllic H, Condamine H, Brûlet $\mathrm{P}$. Pattern of transcription of the homeogene Hox-3.1 in the mouse cmbryo. Genes Dev 1988 ; 2 : 125-35.

10. Kleny M, Mauger A, Sengel P. Early regionalization of the somitic mesoderm as studied by the development of the axial skeleton of the chick embryo. Dev Biol 1972 ; $28: 142-61$.

11. Lance-Jones C. The effect of somite manipulation on the development of motoneuron projection patterns in the embryonic chick hindlimb. Dev Biol 1988 ; 126 : 408-19.

12. Chisaka O, Capecchi MR. Regionally restricted developmental defects resulting from targeted disruption of the mouse homeobox gene Hox-1.5. Nature 1991 ; 350 : 473-9.

13. Lufkin T, Dicrich A, LeMcur M, Mark M, Chambon P. Disruption of the Hox-1.6 homeobox gene results in defects in a region corresponding to its rostral domain of expression. Cell $1991 ; 66$ : 1105-19.

14. McGinnis W, Krumlauf R. Homeobox genes and axial patterning. Cell $1992 ; 68$ : lisme basal de l'organisme. Si l'inactivation de $H o x-3.1$ perturbe ce réseau d'innervation, un grave dérèglement du fonctionnement du tube digestif pourrait alors survenir et fréquemment entraîner la mort.

Certaines souris Hox-3.1 $1^{-1}$ parviennent à l'âge adulte, et mâles et femelles sont féconds. Le phénotype apparent de ces animaux, outre leur petite taille, consiste en certains réflexes anormaux. Chez tous les mutants homozygotes, les doigts des pattes avant sont tellement crispés que les souris ne peuvent rien saisir et marchent sur leurs phalanges (figure 5). A nouveau, l'expression de $H o x-3.1$ dans les cornes ventrales du tube neural suggère la liaison entre une anomalie des motoneurones et les contractions musculaires observées. De plus, le niveau d'expression, élevé dans la région antérieure et diminuant vers la région postérieure, est en relation avec la contraction permanente et très fréquente des membres antérieurs et les contractions plus rares du dos et des membres postérieurs. Une analyse plus fine du système nerveux des souris $H o x-3.1^{-/}$sera nécessaire pour connaître l'origine exacte des différents phénotypes observés.

\section{Détermination antéro-postérieure des cellules embryonnaires par le gène Hox-3.1}

Toutes les souris homozygotes Hox-3.1 $1^{-1-}$ ont montré des transformations de certains segments squelettiques spécifiques en segments plus antérieurs. Des transformations homéotiques similaires sont observées chez la drosophile à la suite de l'inactivation d'un gène homéotique. L'identité de certaines cellules embryonnaires, et non leur position, a été modifiée par la mutation Hox-3.1-lacZ. Les vertèbres $\mathrm{T} 7$ à $\mathrm{L} 1$ dérivent des sous-unités sclérotomes des somites 18 à 25 , qui se forment par compaction du mésoderme paraxial lors du neuvième jour de l'embryogenèse. Des expériences de transplantations ectopiques chez le poulet ont montré que l'identité des sclérotomes était déterminée un peu avant la compaction des somites [10]. Ainsi, lors de son expression précoce

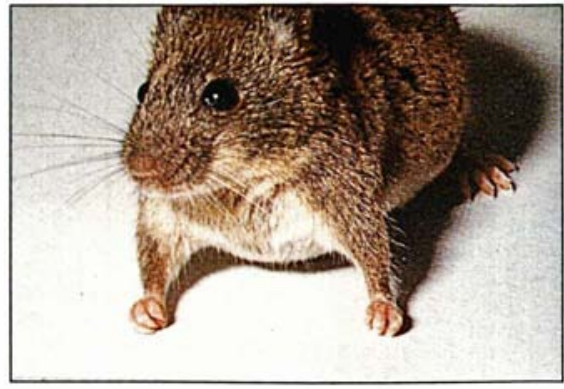

Figure 5. Crispation des membres antérieurs des souris Hox-3.1-1Cette souris ne peut desserrer les doigts des pattes antérieures alors que ceux des pattes postérieures sont relâchés.

entre les jours 8,5 et 9,5, l'homéogène Hox-3.1 apporte certainement aux cellules des informations sur leur position le long de l'axe antéro-postérieur de l'embryon.

Les sternèbres sont dérivées à partir du mésoderme latéral - qui ne montre pas de segmentation morphologique, au contraire du mésoderme paraxial. Le développement d'une sternèbre supplémentaire chez les mutants homozygotes a révélé qu'une transformation homéotique pouvait survenir dans un tissu embryonnaire non segmenté, ce qui n'avait jamais été observé même chez la drosophile. Des transformations homéotiques seraient beaucoup plus difficiles à mettre en évidence dans la moelle épinière, dans laquelle aucune segmentation n'a encore pu être mise en évidence. Ces transformations pourraient survenir au niveau cellulaire et non pas, comme dans le squelette axial, affecter un segment entier. Toutcfois, la détermination spatiale des motoneurones survient également lors de l'expression précoce de $H o x-3.1$, peu avant la compaction des somites [11]. Les deux mutations précédentes d'homéogènes murins par recombinaison homologue, Hox-1.5 et Hox-1.6, n'avaient pas induit de transformations mais, plutôt, la disparition ou la réduction de certaines structures de la région cervicale [12, 13] (voir aussi l'article de M. Mark et al., p. 334 de ce numéro). Ce phénotype peut être dû à la morphogenèse de la région cervicale, qui nécessite des interactions complexes entre le mésoderme, la crête neurale et l'ectoderme neural et superficiel. Les mutations Hox-1.5 et 
Hox-1.6, qui ne semblent affecter que certains types cellulaires, pourraient être incapables d'induire la transformation d'une région d'origine multiplc. Par ailleurs, les gènes Hox-1.5 et Hox-1. 6 sont exprimés dans la région la plus antérieure du domaine d'expression des gènes Hox. La détermination des régions les plus rostrales pourrait être spécifiée d'après un autre programme génétique fondé, par excmple, sur les gènes des familles engrailed ou Distal-less [14]. Alors que les structures touchées par l'inactivation de Hox-3.1 se développent selon un programme génétique modifié, le défaut d'information de position provoqué par l'inactivation de $H o x-1.5$ ou Hox-1.6 pourrait entraîner l'absence complète de programme de développement pour les cellules affectées. Chez la drosophile, également, une mutation dans l'un des homéogènes les plus antérieurs provoque la disparition, et non la transformation, de certaines structures [14]

\section{Summary}

The mouse homeogene Hox-3.1 specifies the identity of some skeletal segments

We have substituted the Hox-3.1 coding sequence by the Escherichia coli lac $Z$ gene through homologous recombination in embryonic stem cells and thus produced null mutant mice. Homozygous mice were born alive but most of them died within a few days. In the trunk region of homozygotes, several skeletal segments were transformed into the likeness of more anterior ones as observed in Drosophila with loss-of-function homeotic mutations. The most obvious transformations were the attachment of the 8th pair of ribs to the sternum and the appearance of a 14th pair of ribs on the first lumbar vertebra. The pattern of $\beta$-galactosidase activity was identical in heterozygotes and homozygotes and reflected faithfully the $H o x-3.1$ expression pattern. Thus the mutation modified the identity, rather than the position, of embryonic cells which would normally express Hox-3.1. 